\title{
Using Radio-Frequency Ablation Electrode in Effective Treatment of Hepatic Rupture in HELLP Syndrome. Case Report and Review of the Literature
}

\author{
Honorata Stadnik* and Tomasz Kościński
}

Department of General, Endocrine and Oncologic Surgery, Poznań University of Medical Sciences, Poland

\begin{abstract}
Spontaneous hepatic rupture constitutes very rare complication of pregnancy and is associated with an increased risk of mortality. The patient presented at 30 weeks of gestation with HELLP syndrome developed life threatening hepatic rupture. She underwent an emergent cesarean delivery followed by exploration of her abdomen by hepatobiliary surgeons. An expanding hematoma with rupture of the right and left lobe of the liver was successfully treated with radiofrequency ablation electrode. The patient was discharged home in good condition 13 days after surgery. Quick diagnosis, multidisciplinary approach and radio-frequency ablation help to achieve an excellent clinical outcome in a patient with hepatic rupture from HELLP syndrome.
\end{abstract}

Keywords: HELLP syndrome; Hepatic rupture

\section{Introduction}

Spontaneous hepatic rupture constitutes very rare complication of pregnancy and is associated with an increased risk of mortality. First case of a non-traumatic liver rupture in pregnant was reported in 1844 by Abercrombie [1]. The incidence of spontaneous hepatic rupture cases in pregnant women ranges from 1 to 45000 to 1 to 225000 of deliveries [2]. The condition may be related with preeclampsia and eclampsia, but in particular it affects women with HELLP syndrome.

The acronym HELLP was coined by L. Weinstein in 1982, and derives from the first letters of triad of symptoms observed in some patients diagnosed with preeclampsia $(\mathrm{H}$ - hemolysis, EL - elevated liver enzymes, LP - low platelets count). Initially, the syndrome was treated as severe form of preeclampsia or its complication, but nowadays it is diagnosed as distinct entity. HELLP syndrome occurs in $0.5 \%$ to $0.9 \%$ of all pregnancies and in 10 to $20 \%$ of cases with preeclampsia. In $70 \%$ of the cases it is recognized during pregnancy, mainly in the third trimester, and in the rest of the cases diagnosis is made during post-partum, what does not mean that the syndrome has not been developing during pregnancy. After delivery the symptoms occur most frequently during the first $48 \mathrm{~h}$, but they may appear up to sixth week of postpartum. HELLP syndrome pathogenesis is not fully understood [3-6]. Endothelial cell damage, platelets activation, adhesion and aggregation, thromboxane $\mathrm{A}_{2}$ and serotonin release, local vasoconstriction of blood vessels, and fibrin deposition can contribute to this complication. Red blood cells are destroyed while passing through small vessels with damaged endothelium where fibrin deposits are localized what results in microangiopathic hemolytic anemia [3].

Elevated liver transaminases activity is the effect of hepatic vascular occlusion consequently leading to hypoxia and necrosis of hepatocytes. Subcapsular liver hematoma and hepatic rupture occur in less than $5 \%$ of the HELLP cases. Thrombocytopenia, which also appears in the syndrome, intensifies the symptoms of bleeding and hinders to achieve hemostasis of bleeding organ. Herein, the case of spontaneous hepatic rupture in pregnant is reported and discussed.

\section{Case Report}

A 29-year-old primipara was admitted at 30 weeks of gestation to delivery ward, being transferred from emergency department at a general hospital, for the suspected preeclampsia. Since $14^{\text {th }}$ week of gestation she has been effectively treated for hypertension. Initially, she received methyldopa $250 \mathrm{mg}$ twice daily and since $26^{\text {th }}$ week of gestation $250 \mathrm{mg}$ three times daily. The patient's complaints were severe chest pain and high blood pressure reaching values of 165/100 $\mathrm{mmHg}$. Myocardial infarction and acute coronary syndrome has been ruled out in an electrocardiogram and serial measurement of cardiac troponin T. Furthermore, proteinuria, elevated levels of ALT $163 \mathrm{U} / \mathrm{L}$, AST $152 \mathrm{U} / \mathrm{L}, \mathrm{LDH} 313$; Hgb $7 \mathrm{mmol} / \mathrm{L}$, and decreased platelets count to $109 \mathrm{G} / \mathrm{L}$ were found. The patient was transported to a specialized delivery ward. No abnormalities in abdominal and fetal ultrasound were found. Treatment of high blood pressure was undertaken which succeeded in blood pressure lowering to $120-130 / 65-70 \mathrm{mmHg}$. In the following days the pain in the chest slightly ceased. Normal blood pressure was maintained. Laboratory findings revealed intensifying hemolytic anemia, decrease in platelets count, a continuous increase in liver transaminases levels and $\mathrm{LDH}$, which enabled the diagnosis of HELLP. On the fifth day of hospitalization, in the evening, patient's state deteriorated. The patient complained from very severe chest pain. Blood pressure reached $180 / 100 \mathrm{mmHg}$. The other findings were as follows: Hgb $5.2 \mathrm{mmol} / \mathrm{L}$, platelets count $46 \mathrm{G} / \mathrm{L}$, AST 209, ALT 236, direct bilirubin $0.45 \mathrm{mg} / \mathrm{dL}$. In the night hours ultrasounds revealed free fluid in peritoneal cavity and subcapsular hematoma of liver. The decision to terminate the pregnancy by cesarean section was made. The $10 \mathrm{mg}$ of dexamethasone and the ampoule of magnesium sulphate were administered.

At laparotomy altered blood in the peritoneal cavity was found. Female fetus of birth weight of $1370 \mathrm{~g}$ was extracted. Subsequently, the midline incision was extended above the umbilicus. A ruptured subcapsular hematoma of right lobe of the liver was found. Hemostatic sutures and bipolar forceps were used in an attempt to control bleeding from the liver. As the procedures were not effective, compression compresses between the dome of the diaphragm and the right lobe of the liver were assumed. The abdominal cavity was closed. A few hours later, in the morning, 2 liters of bloody content appeared in the drains.

*Corresponding author: Honorata Stadnik, Department of General, Endocrine and Oncologic Surgery, Poznań University of Medical Sciences, Poland, Tel: +48 6185460 00; E-mail: honoratastadnik@wp.pl

Received November 14, 2017; Accepted December 22, 2017; Published December 27, 2017

Citation: Stadnik H, Kościński T (2017) Using Radio-Frequency Ablation Electrode in Effective Treatment of Hepatic Rupture in HELLP Syndrome. Case Report and Review of the Literature. J Clin Case Rep 7: 1053. doi: 10.4172/2165 7920.10001053

Copyright: @ 2017 Stadnik H, et al. This is an open-access article distributed under the terms of the Creative Commons Attribution License, which permits unrestricted use, distribution, and reproduction in any medium, provided the original author and source are credited. 
Citation: Stadnik H, Kościński T (2017) Using Radio-Frequency Ablation Electrode in Effective Treatment of Hepatic Rupture in HELLP Syndrome. Case Report and Review of the Literature. J Clin Case Rep 7: 1053. doi: 10.4172/2165-7920.10001053

Page 2 of 4

The patient was transported to an operating room of the Department of General, Endocrine and Oncologic Surgery. Midline incision was extended to the xiphoid process. After removing clots, massive bleeding from the surface of the entire right hepatic lobe with a capsule rupture, VII segment of the liver tear to a depth of $3 \mathrm{~cm}$, and a subcapsular hematoma in III segment of the left lobe of the liver were found. Bleeding was controlled effectively by radiofrequency ablation of liver parenchyma with radio-frequency ablation electrode. The torn capsule was removed. The fragments of liver parenchyma were collected for histopathological examination. Complete hemostasis was achieved in the area of the ruptured segment VII and the entire surface of the right lobe of the liver. Subcapsular hematoma of the III liver segment was ablated with the same method, so that it was removed, and the integrity of the capsule with the liver parenchyma was achieved. The peritoneal cavity was flushed with saline, and drains were placed under the dome of the diaphragm and into the pouch of Douglas. In the postoperative course within a few days platelets count has standardized to normal levels, which was maintained until the end of hospitalization, similar to LDH and direct bilirubin levels decrease. Proteinuria subsided. Hepatic transaminases increased to few thousand during the first day after surgery as a result of the method used to control of bleeding and subsequently decreased in several days to the normal range.

The catheter was removed from the pouch of Douglas on the second day after the surgery. Drain from the liver area was maintained longer, allowing the evacuation of exudate after ablation of the liver surface, which prevented the formation of supdiaphragmatic and perihepatic reservoirs. The drain was removed in $6^{\text {th }}$ day after surgery.

A further period of postoperative course was uneventful. The patient was discharged home on $13^{\text {th }}$ postoperative day in good general condition. Ten months after delivery the mother and child are doing well. The girl is developing normally. Mother's imaging studies with ultrasounds and CT did not identify any tanks or morphological changes in the liver.

\section{Discussion}

Less than 200 cases of spontaneous rupture of the liver associated with HELLP syndrome, preeclampsia and eclampsia have been reported in the English literature and analyzed so far [7]. Most of the descriptions is very eclectic and focuses on the complications and obstetric surgical interventions rather than on the evolution of syndrome and accompanying clinical symptoms, laboratory and imaging diagnostics, which could help to find the answer to the question how to effectively treat and prevent the development of subcapsular hematoma and rupture of the liver.

Spontaneous rupture of subcapsular hematoma and liver parenchyma occurs in 1 in 67,000 births and 1 in 2,000 pregnant women with preeclampsia, eclampsia and HELLP syndrome [8]. In the 1990-2010 years 180 such cases were described in European literature. In $10 \%$ they were related to the rise of subcapsular hematoma without rupture of the liver, in 162 cases there was a rupture of the liver capsule and its parenchyma. In one case it concerned a patient with eclampsia without HELLP syndrome development, in 12 cases HEEH syndrome, and 167 pregnant women had symptoms of a fully developed HELLP syndrome. Mean age at diagnosis was $30.9 \pm 5$ years, $86.5 \%$ were above 25 years of age, and $52.1 \%$ aged $28-35$ years [9].

The most common localization of hematoma and bleeding from a liver is the right lobe [8-10]. It is observed in $77 \%$ of the cases. Hematoma in two lobes occurs in $21 \%$ of patients. It is difficult to assume, based on data from literature, whether hematoma only in left hepatic lobe does occur. None of those cases were described, and in $2 \%$ of reported cases the localization has not been shown or was impossible to determine due to big damage of the liver.

The falciform ligament of the liver is a strong band of connective tissue which connects the liver with the diaphragm and divides the liver on the right and left lobe. At this point, Glisson capsule strongly adheres to the liver parenchyma and acts as a mechanical barrier to the spread of the hematoma. Based on the literature and our own experience, it is possible to assume unequivocally that due to unknown causes damage to the vascular endothelium of the hepatic vessels occurs in the right lobe and a transition to the left lobe is the result of the development of a hematoma in the case of delayed diagnosis and treatment. This situation relates to the case described in this article where initially hematoma and hemorrhage concerned only the right hepatic lobe of the liver and after unsuccessful supply eight hours later disseminated the border of the falciform ligament and detached the capsule in the part of III segment of the left hepatic lobe.

Such confirmation can be found in the results of the histopathological examination of tissues taken from both lobes of the liver. In the right lobe the lesion of endothelium of the liver parenchymal vessels, intravascular deposition of fibrin, and periportal bleeding were assumed, while in the specimen taken from the left lobe there were no abnormalities.

Massive bleeding from the liver can be directly life-threatening condition. Failure to start any treatment leads to the death of the patient. Spontaneous rupture of the liver in pregnant women is burdened with high mortality rates. With the development of surgical techniques and anaesteshiological treatment these results have greatly improved in recent years. Until 1970 the mortality of pregnant women with bleeding from the liver without taking a surgical action reached $100 \%$ [11]. Ten years later it decreased to $77 \%$ [12], and in the late 90 's was $39 \%$ if a surgical intervention was used [13]. In the analysis of 180 cases from 1990 to 2010 , in the first decade mortality reached $28.9 \%$, and significantly decreased in $2000-2010$ to $16.4 \%$ [9].

In the matter of treatment in the analyzed material in 18 cases (10\%) embolization of the hepatic artery was sufficiently effective to stop the bleeding. Hepatic artery ligation was used in case of 12 patients, of which 2 patients died. Liver transplantation was performed in 13 women in postpartum period. One of them died in the first of two analyzed decades. Organ transplantation is necessary in cases of progressive necrosis of hepatocytes in whole liver, which leads to acute organ failure and in the case of such a liver damage which cannot be provided by available surgical techniques [5].

In the largest proportion of $58.3 \%$ of the cases combined techniques were applied. Surgical resection of liver parenchyma with hepatic artery ligation or embolization was effective in $75 \%$. Comparing the results of the first and second decades attention can be paid on the increase in the percentage of method efficacy from $66 \%$ to $84 \%$, which is associated with the improvement of surgical techniques and the development of power tools which are used nowadays. A number of effective methods of surgical control of bleeding from the liver were described. Among them were using of abdominal tampo with compresses in the area between the liver capsule and diaphragm, hemostatic sutures, ligation of the hepatic artery, classical resection using the harmonic scalpel and the water scalpel, achieving hemostasis with argon plasma coagulation [14], tissue adhesives, or hemostatic materials. So far there were no reports of the technique presented in this article, which is the control of bleeding from the liver using radio-frequency ablation electrode. Electrode, introduced for use as a tool for thermal radiofrequency 
Citation: Stadnik H, Kościński T (2017) Using Radio-Frequency Ablation Electrode in Effective Treatment of Hepatic Rupture in HELLP Syndrome. Case Report and Review of the Literature. J Clin Case Rep 7: 1053. doi: 10.4172/2165-7920.10001053

Page 3 of 4

ablation of focal cancer lesions in the liver and other organs, quickly found application in liver resection surgeries. It enables performing anatomical and non-anatomical resections in liver. It allows achieving good hemostasis in resected liver section parenchyma and a good supply of the bile ducts, preventing complication in the form of biliary fistula. Certainly, this method can be considered as a very effective to stop bleeding both from a bleeding surface of subcapsular area of the liver and a ruptured liver parenchyma section, while maintaining the integrity of the organ and avoiding resection of part of the liver.

In the analyzed group of 180 patients, 23 required adjunctive therapy in the intensive care unit, catecholamines, the use of recombinant factor VIIa, considered as effective in stopping of bleeding, transfusions of plasma and platelet concentrate [9]. Taking into consideration still high mortality when HELLP syndrome is complicated by spontaneous bleeding from the liver, there were attempts to analyze the moment of rising signs and laboratory exponents at which bleeding occurs. Darby et al based on well-described 87 cases reported in the literature within 1976 to 2011, have found that bleeding from the liver occurs in the smallest proportion (13.8\%) in pregnant in third class of HELLP syndrome with platelet count between 100000 and 150000 , moderately elevated liver transaminases, and severe hemolysis with $\mathrm{LDH}>600$ IU/L. In the first class, in which the number of platelets drops to less than 50000 the complications occurred among 18 patients (20.7\%). The largest group consisted of 24 (27.6\%) patients classified into the second class of syndrome at the moment of diagnosis of the bleeding. The weak point of the analysis was the absence of the possibility to classify the 33 pregnant women to any of the classes due to lack of sufficient data. Certainly, it can be claimed that the risk of changes in the liver in HELLP syndrome increases with the escalation of liver transaminases and a decrease in platelets. It is not possible to conclude univocally that in patients in first class of HELLP with platelets less than 50000 bleeding occurs the mostly commonly, although it seemed to be a logical consequence. The answer to the question whether this logical reasoning does not apply to the HELLP syndrome cannot be found in the literature [7]. A diagnostic imaging performed early enough has a very important role in the prevention of HELLP syndrome complications. In the material of Derby subcapsular hematoma and bleeding from the liver were found by laparotomy in $32 \%$ of cases, which in comparison with other reports constitutes small percentage. In $20 \%$ of cases the diagnosis was able to be made based on abdominal ultrasonography, in $30 \%$ based on CT, in $8 \%$ using both methods, and in $2 \%$ based on the post-mortem examination. Imaging studies, however, were made too late, as bleeding required already surgical intervention in all patients. The predominant symptom, reported by more than $80 \%$ of patients, was epigastric pain and pain in the right upper quadrant. Of course, there have been reports of atypical symptoms and HELLP syndrome development, which lead to cardiac tamponade, pulmonary embolism, or severe pain in the chest mimicking myocardial ischemia. On the basis of the case described in this paper, in which there was lack of diagnostic imaging of the liver, and based on literature data, it seems that systematic monitoring of patients with HELLP syndrome using minimally invasive abdominal ultrasound would allow for early diagnosis of subcapsular hematoma and effective intervention. The latter reduces the mortality rate of mothers and maybe also fetuses, without exposing them to hypovolemic shock associated with massive bleeding.

In the study group there was a large proportion of fetal deaths $24.1 \%$ and $3.4 \%$ of neonatal deaths at an average week of the pregnancy $33 \pm 5.3$. Arauho et al. in paper analyzing 10 cases of spontaneous rupture of the liver in 1990-2002 in Brazil described the 7 deaths of fetuses and one of newborn immediately after birth [2].
HELLP syndrome usually develops turbulent, within a few days or even several hours. It is a systemic disease with constant progression of the symptoms. Treatment is very difficult, and its establishment requires large prospective studies. The only effective way to eliminate the symptoms is labor, suggested by many authors as the only appropriate treatment preceded by the administration of corticosteroids in a group of pregnant women under 34 weeks of pregnancy.

Runnard et al. conducted a study on a group of 31 pregnant women with HELLP syndrome under 30 gestational weeks with administering prednisolone at a dose of $50 \mathrm{mg}$ every $12 \mathrm{~h}$ in the first group of 15 patients and placebo in the second group of sixteen people. In both groups the time from the beginning of the test to delivery was similar (6.8 days in the first vs 8 days in the second). Perinatal mortality was $20 \%$ in the first group and $25 \%$ in the second. In the second group in three cases the subcapsular hematoma and bleeding from the liver occurred and one patient died. Visser and Wallenburg presented a group of 128 pregnant women with HELLP syndrome under 34 gestational weeks who were treated with transfusions of plasma and vasodilators under constant monitoring of blood pressure. Twenty-two pregnant women delivered within $48 \mathrm{~h}$; the remaining 106 patients' childbirth was managed to be postponed to the mean of 15 days; fifty-five pregnant women delivered in 3 weeks after the diagnosis of HELLP. There were no patient deaths, and 11 of women had bleeding complications. Perinatal mortality was $14 \%$. These results were comparable to the same number of patients in a group of pregnant women with pre-eclampsia syndrome without developed HELLP syndrome [15].

Pampus et al. recommended bed rest, antihypertensive drugs, and limiting salt intake as a treatment in a group of 41 pregnant women with HELLP syndrome under 35 weeks of gestation. Fourteen patients (34\%) delivered within 24 hours; in the rest 27 pregnant women the delivery was managed to be delayed to the average of three days. There were no deaths among pregnant women and perinatal mortality was $25 \%[16]$.

In all aforementioned studies there is no detailed analysis of patients' class of the HELLP syndrome. Martin et al. postulate the treatment of pregnant in the first and second class of HELLP with the dose of $10 \mathrm{mg}$ of dexamethasone every $12 \mathrm{~h}$, monitoring in intensive care units, and systematic assessment of liver enzymes, $\mathrm{LDH}$, platelets and bilirubin. In a case of decrease in platelet count below 40,000 they postulate intubation and mechanical ventilation. In a case of pregnant women in the third class of syndrome, complicated by pre-eclampsia, abdominal pain, hypertension, cardio-pulmonary and nephrological disturbances the same procedures as in class 1 and 2 are recommended. In the group with uncomplicated course astute observation with blood pressure measurement, and laboratory tests are suggested. In the group of patients under 24 weeks of gestation early termination of pregnancy is postulated; between 24 and 34 weeks postponement of childbirth by 24 to $48 \mathrm{~h}$ in an aim to treat with corticosteroids to achieve mutual benefit of both mother and fetus. Over 34 weeks of pregnancy postponement of delivery to 6-12 h after administration of corticosteroids [17].

\section{Conclusion}

As far as the effective treatment allowing for the reversal of HELLP syndrome will be found, Martin's postulates are the only currently recognized as appropriate. When expanded to include a thorough systematic imaging of the abdomen, they may allow the creation of standards of management of HELLP syndrome cases complicated by subcapsular hematoma of the liver.

Patients with HELLP syndrome should be hospitalized in centers 
Citation: Stadnik H, Kościński T (2017) Using Radio-Frequency Ablation Electrode in Effective Treatment of Hepatic Rupture in HELLP Syndrome. Case Report and Review of the Literature. J Clin Case Rep 7: 1053. doi: 10.4172/2165-7920.10001053

with extensive experience in the field of pathology of pregnancy with a good and properly close surgical facility experienced in liver surgery. In cases of liver spontaneous rupture in pregnant effective way to stop bleeding is liver ablation using radio-frequency ablation electrode.

The progress of medicine and its possibilities, also in the field of neonatology, allows the births of children with lower and lower birth weight in the earlier stage of fetal life. Pathomechanisms of HELLP syndrome which results in such a high perinatal mortality is not exactly known. The decision to interrupt the immature pregnancy, weighing in the balance life of the mother and child, remains difficult. It is why so important are prospective multicenter studies to determine the precise pathogenesis of HELLP and its effects on the fetus and methods of disease treatment.

\section{References}

1. Abercrombie J (1844) Hemorrhage of the liver. London Medical Gazette 34 792-794.

2. Araujo AC, Leao MD, Nobrega MH, Bezerra PF, Pereira FV, et al. (2006) Characteristics and treatment of hepatic rupture caused by HELLP syndrome. Am J Obstet Gynecol 195: 129-133.

3. Padden MO (1999) HELLP syndrome: Recognition and perinatal management Am Fam Physician 60: 829-836, 839.

4. Haram K, Svendsen E, Abildgaard U (2009) The HELLP syndrome: Clinica issues and management. A Review BMC Preg Childbirth 9: 8.

5. Mihu D, Costin N, Mihu CM, Seicean A, Ciortea R (2007) HELLP syndrome- a multisystemic disorder. J Gastrointestinal Liver Dis 16: 419-424.

6. Miguelote RF, Costa V, Vivas J, Gonzaga L, Menezes CA (2009) Postpartum spontaneous rupture of a liver hematoma associated with preeclampsia and HELLP syndrome. Arch Gynecol Obstet 279: 923-926.

7. Darby M, Martin JN, Mitchell SQ, Owens MY, Wallace K (2013) Using case reports to determine when liver bleeding occurs during disease progression in HELLP syndrome. International Journal of Gynaecology and Obstetrics: The official organ of the International Federation of Gynaecology and Obstetrics 123: 7-9.

8. Pavlis T, Aloizos S, Aravosita P, Mystakelli C, Petrochilou D, et al. (2009) Diagnosis and surgical management of spontaneous hepatic rupture associated with HELLP syndrome. J Surg Educ 66: 163-167.

9. Vigil-De Gracia P, Ortega-Paz L (2012) Pre-eclampsia/eclampsia and hepatic rupture. International Journal of Gynaecology and Obstetrics: The official organ of the International Federation of Gynaecology and Obstetrics 118: 186-189.

10. Poo JL, Gongora J (2006) Hepatic hematoma and hepatic rupture in pregnancy. Ann Hepatol 5: 224-226.

11. Heller TD, Goldfarb JP (1986) Spontaneous rupture of the liver during pregnancy. A case report and review of the literature. New York state Journal of Medicine 86: 314-316.

12. Henny CP, Lim AE, Brummelkamp WH, Buller HR, Ten Cate JW (1983) A review of the importance of acute multidisciplinary treatment following spontaneous rupture of the liver capsule during pregnancy. Surg Gynecol Obstetrics 156: 593-598.

13. Reck T, Bussenius-Kammerer M, Ott R, Muller V, Beinder E, et al. (2001) Surgical treatment of HELLP syndrome-associated liver rupture- An update. European Journal of Obstetrics, Gynecology and Reproductive Biology 99: 57-65.

14. Shrivastava VK, Imagawa D, Wing DA (2006) Argon beam coagulator for treatment of hepatic rupture with hemolysis, elevated liver enzymes, low platelets (HELLP) syndrome. Obstet Gynecol 107: 525-526.

15. Visser W, Wallenburg HC (1995) Temporising management of severe preeclampsia with and without the HELLP syndrome. BJOG 102: 111-117.

16. Van Pampus MG, Wolf H, Westenberg SM, Van der Post JA, Bonsel GJ, et al. (1998) Maternal and perinatal outcome after expectant management of the HELLP syndrome compared with pre-eclampsia without HELLP syndrome. European Journal of Obstetrics, Gynecology, and Reproductive Biology 76 $31-36$

17. Martin JN, Rose $\mathrm{CH}$, Briery $\mathrm{CM}$ (2006) Understanding and managing HELLP syndrome: The integral role of aggressive glucocorticoids for mother and child Am J Obstetrics Gynecol 195: 914-934. 